\title{
A QoS Constraints Location-based Services Selection Model and Algorithm under Mobile Internet Environment
}

\author{
Mingjun Xin, Tianle Jiang and Rui Zhang \\ School of Computer Science and Engineering, Shanghai University, \\ Shanghai 200444, China \\ xinmj@shu.edu.cn,yuleharry@shu.edu.cn,ruizhang@shu.edu.cn
}

\begin{abstract}
With the rapid development of communication technology and mobile Internet, selecting from mass Location-based Services to meet the needs for different users becomes the research hot spot under mobile Internet environment. First of all, it starts from QoS constraints as well as user preferences in this paper, and then describes a mobile service selection framework based on QoS constraints and user preferences. Furthermore, a User Preferences Database is built in modeling user preferences by Similarity Formula. Users' feedback is adopted to update User Preferences Database, which makes the combination between QoS constraints and user preferences more compact. Then, a LBS selection model and algorithm are proposed separately, which considers the QoS attributes and user preferences. Finally, it takes Online Learning Resources Platform (OLRP) based on mobile terminals as a sample, and designs a mobile course resources selection system on campus. The results show that the proposed algorithm is precision and effective in solving the problem of service selection to support Location-based Services personalization.
\end{abstract}

Keywords: Location-based Services (LBS), Service selection, Quality of Service (QoS), User preferences

\section{Introduction}

As the rapid development of mobile Internet and communication technology, there are large scales of services and applications on the network. Internet of Things (IoT), Location-based Services (LBS) and Cloud Computing become more and more popular applications and areas under mobile Internet environment. But how to choose appropriate service turns to be the key problems for the development of those technologies. So service selection [1] is investigated quite intensively in recent years.

LBS can be defined as information services accessible with mobile devices through the mobile network and utilizing the ability to make use of the location of the mobile device. Although a lot of effort has been spent on LBS selection [2], most of those methods [3] concentrate on the functional characteristics of service. Academia and industry pay more and more attention to service selection [5] based on quality of service (QoS), which is the important part of nonfunctional characteristics. There are some achievements in QoS-based service selection [6], but there are no effective methods focusing on both QoS attributes and user preferences. So a service selection [7] model and algorithm which considers both QoS constraints and user preferences are put forward. And it will utilize users' feedback to update User Preferences Database, which stores the users' preferences and action [8]. 


\section{Modeling of Location-based Services Selection}

\subsection{Framework of Service Selection}

In this paper, it presents an algorithm of service selection which combines QoS and user preferences. At the same time, it gives the initialization and update methods of user preferences in User Preferences Database. To solve users' mass problems and coldstart problem, it uses the classification description technology, which classifies the similar users and computes the user group preferences. Also it considers the relationship between personal and user group when using user preferences, so it proposes a method to compute this kind of relationship.

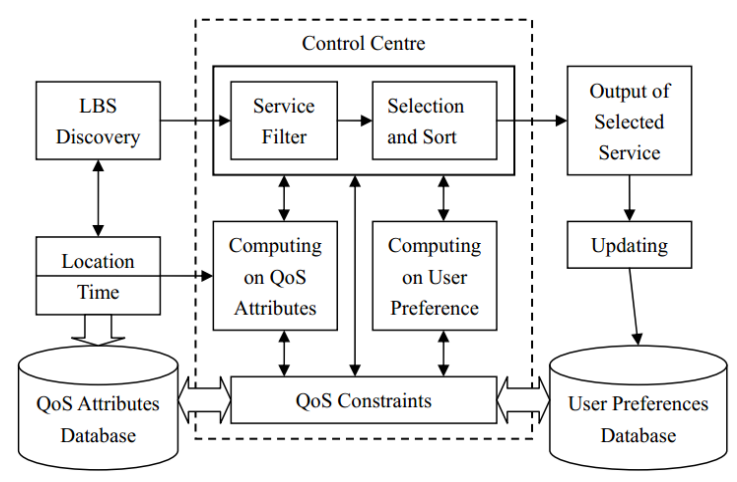

Figure 1. Framework of LBS Selection

A framework of Location-based Services selection model under mobile Internet environment is shown in Figure 1, which contains three modules and two databases. Three modules in Figure 1 are Input Module, Control Module and Output and Feedback Module. Two databases are QoS Attributes Database and User Preferences Database.

\subsection{Modeling of QoS Attributes}

QoS [9] is "characterized by the combined aspects of service support performance, service operability performance, service ability performance, service integrity and other factors specific for each service." Some dimensions are set to meet the needs of LSB selection according to this definition. Here it gives definition of QoS [10]:

Definition 1: QoS: 〈Cost; Duration; Time; Location; Usability; Reliability>

Cost is the price if user wants to obtain the service. Cost is a general concept in various kinds of services.

Duration means the time spent from service request submitting to service ending. The index may have very large difference to different types of services.

Time means when the services take place. This is a special aspect in Location-based Services. Time is an important factor in Service Filter. Users can make use of this condition to choose suitable services.

Location is also a special aspect in Location-based Services for the space distance is considered by many users. And it is a significant difference from traditional services.

Usability means the probability of the correct response to service request.

Reliability means the security of service which is reflecting the degree of falsity. 
In this paper, it doesn't focus on how to obtain the QoS attributes, while it supposes that QoS Attributes Database has stored that information. The data in the database have different units and should be quantified firstly. The quantification of QoS makes the QoS attributes have the same measuring standard. Before the quantification it should check what kind of QoS attributes is. So it takes formula 1 to quantify the positive QoS attributes (Usability; Reliability), while formula 2 to the negative (Cost; Duration).

$$
\begin{aligned}
& q_{i}= \begin{cases}\frac{Q o S_{i}-Q o S_{\text {min }}}{Q o S_{\text {max }}-Q o S_{\text {min }}} & Q o S_{\text {max }}-Q o S_{\text {min }} \neq 0 \\
1 & Q o S_{\text {max }}-Q o S_{\text {min }}=0\end{cases} \\
& q_{i}= \begin{cases}\frac{Q o S_{\text {max }}-Q o S_{i}}{Q o S_{\text {max }}-Q o S_{\text {min }}} & Q o S_{\text {max }}-Q o S_{\text {min }} \neq 0 \\
1 & Q o S_{\text {max }}-Q o S_{\text {min }}=0\end{cases}
\end{aligned}
$$

In formula 1 and 2, $q_{i}$ means quantified QoS attributes and $Q o S_{i}$ means original QoS attributes. $Q o S_{\max }$ and $Q o S_{\min }$ mean maximum and minimum items in one dimension of QoS attributes. QoS attributes can be easily quantified using these formulas. So LSB can be selected though quantified QoS attributes under mobile Internet environment.

The formula 1 and 2 solves the modeling of Usability, Reliability, Duration and Cost. While Location, which is a symbol of Location-based Services, need to be modeled with different method.

First a conception of distance between user and LBS should be defined:

Definition 2: Distance: The space distance from user to Location-based Services.

Users may have different distance conception in space distance under different context. Here Acceptive Distance is set as users' default space distance:

Definition 3: Acceptive Distance: Users' default space distance in LBS selection. It acts as a standard distance, and if the actual distance is larger than Acceptive Distance then this LBS has smaller attraction to user.

To quantify this attraction in service selection, formula 3 is presented:

$$
Q o S=\frac{D_{\alpha}}{D_{\beta}}
$$

Here $D_{\alpha}$ means the actual distance from user to LBS, and $D_{\beta}$ means the Acceptive Distance set by user. It use one kilometer as the default value. The actual distance can be easily got under mobile Internet. So QoS attribute of Location can be gotten by Service Selection System. The initial value in the formula 3 hasn't been quantified. Considering this kind of QoS attribute is negative, so quantified QoS attributes are got using formula 2.

\subsection{Modeling of User Preferences}

Bruce Krulwich [11] classifies the entire Americans into 62 groups according to the demography attributes. Demography attributes contain age, gender, job, education, residence, nationality and nation. This paper uses that method to classify users according to their registration data, which includes school, major, age, gender, job and nation. Just for different groups have different preferences to QoS attributes, in this paper, it takes the user group's preference [12] as initial value for a new user to solve the cold-start problem. 
In modeling of user preferences [13], formula 4 presents the basic method to determine the user group which one user belongs to. In this formula, $\operatorname{sim}(a, b)$ defines the similarity between user and user group. This Cosine Similarity Formula calculates the matching degree between user and user group.

$$
\operatorname{sim}(a, b)=\cos (\vec{a}, \vec{b})=\frac{\vec{a} \cdot \vec{b}}{\|\vec{a}\| \cdot\|\vec{b}\|}
$$

Getting which user group does one user belong to, then it can set user preferences as user group preferences, that is $W_{U}=W_{G}\left[G R O U P_{b}\right]$. Here $W_{G}\left[G R O U P_{b}\right]$ is user group preferences and $b$ means user belongs to user group $b$, and $W_{U}$ is user preferences. When user has some choices that means there are more than $R I$ records for this user in User Preferences Database, user preferences is not only effected by user group, but also by personal user preferences. So formula 5 is used to calculate user preferences.

$$
W_{U}=\alpha_{3} W_{G}\left[G R O U P_{b}\right]+\beta_{3} W_{P}
$$

In this formula, $W_{P}$ is personal user preferences. As user has more and more choices, assuming if there are more than $R 2$ records in User Preferences Database, user group preferences will have negative effect on calculation user preferences. So $W_{U}=W_{P}$.

In formula 5, the value of $\alpha_{3}$ and $\beta_{3}$ will affect the computing process. It assumes there are $R$ records in User Preferences Database, and $R$ is larger than $R 1$ but smaller than $R 2$. So it presents formula 6 and 7 to calculate the value of $\alpha_{3}$ and $\beta_{3}$.

$$
\begin{aligned}
& \alpha_{3}=(R 2-R) /(R 2-R 1) \\
& \beta_{3}=(R-R 1) /(R 2-R 1)
\end{aligned}
$$

In formula 6 and 7, as users have more and more their own choices, user preferences will more focus on personal user preferences.

In this modeling part, first it uses formula 4 to determine user group at first. As users have their choices after the system selects Location-based Services, the Service Selection System may check whether the personal user preferences and user group preferences have big discrepancy. This updating process of user preferences will be discussed in next part.

Then formula 8 shows how to calculate user preferences.

$$
W_{U}=\left\{\begin{array}{cc}
W_{G}\left[\text { GROUP }_{b}\right] & R<R 1 \\
\frac{R 2-R}{R 2-R 1} W_{G}\left[\text { GROUP }_{b}\right]+\frac{R-R 1}{R 2-R 1} W_{P} & R 1 \leq R \leq R 2 \\
W_{P} & R>R 2
\end{array}\right.
$$

\section{Algorithm of LBS Selection}

In this selection, it gives a detailed description of the algorithm of service selection, which includes the selection part and the updating part. 


\subsection{Description of LBS Selection}

In this paper, it sorts the LBS by different kinds of QoS attributes. It supposes the QoS attributes have $m$ dimension $\left(q_{1}, q_{2} \ldots q_{m}\right)$, and $q_{j}(j=1,2 \ldots m)$ means the $j$-th QoS attributes. $L B S$ Discovery will get $n$ services $\left(S_{1}, S_{2} \ldots S_{n}\right)$, and $S_{i}(i=1,2 \ldots n)$ means the service $i$. The QoS attributes of service $i$ are stored in QoS Attributes Database. So it supposes there is a $n \times m$ matrix $Q$. Computing on $Q o S$ Attributes in figure 1 can quantify this matrix $Q$, so the elements in $Q$ is defined between 0 to 1 .

It uses skyline approaches to optimize the matrix by pruning some services in this paper. Then, it supposes there are $N S$ services left after using skyline optimization.

For one user, there are $W_{G}[t]$ and $W_{P}$ in User Preferences Database. $W_{P}$ means the personal user preferences, and $W_{G}[t]$ means the user group preferences. The $t(t=1,2 \ldots H)$ in $W_{G}[t]$ means user belongs to user group $t$., User preferences $W_{U}$ is calculated in formula 8 .

The weight of user preferences is initialized that all the weight has same value, that is $W_{U}[j]=1 / m(j=1,2 \ldots m)$.

To sort the LBS, the Unified QoS Attributes is calculated in formula 9.

$$
Q o S_{i}=\sum_{j=1}^{m} W_{U}[j] \times Q[i][j](i=1,2 \ldots N S)
$$

Definition 4: $Q o S_{i}$ is the Unified $Q o S$ Attributes of service $i . Q[i][j]$ is an element in $Q$ and the $i$ means service $i$, while $j$ is the $j$-th QoS attribute.

When it calculates all the Unified QoS Attributes $\left(Q o S_{1}, Q o S_{2} \ldots Q o S_{N S}\right)$ which act as selection condition, it can select and sort those services. Finally $N T$ services are outputted from those $N S$ services.

\subsection{Pseudo Code of Algorithm}

The whole process is divided into two parts. One is the LSB selection algorithm in (a). It contains computations of user group and weight of user preferences, and also contains skyline approaches and computing of Unified QoS Attributes.

\begin{tabular}{|c|c|}
\hline \multicolumn{2}{|c|}{$\begin{array}{l}\text { (a) Service Selection Algorithm } \\
\text { Input: user ID, } n \text { services outputted by LBS Discovery } \\
\text { Output: } T S \text { services }\end{array}$} \\
\hline $\begin{array}{l}\text { 1. while }(b<H) \\
\qquad \operatorname{sim}(a, b)=\cos (\vec{a}, \vec{b})=\frac{\vec{a} \cdot \vec{b}}{\|\vec{a}\| \cdot\|\vec{b}\|} \\
\text { 2.if }\left(\operatorname{sim}\left(a, G_{R O U P}\right)=\max \{\operatorname{sim}(a, b) ; b=1,2 \ldots H\}\right) \\
\quad a \in \operatorname{GROUP}_{b} \\
\text { //user } a \text { belongs to user group GROUP } \\
\text { 3. if (user is new) } \\
\quad W_{U}=W_{G}[\text { GROUP } \\
\text { 4. else }\{\end{array}$ & $\begin{array}{l}n: \text { the number of services in } L B S \text { Discovery } \\
T S: \text { the number of selected services } \\
a: \text { One user who uses the Service Selection } \\
\text { System } \\
b: \text { One user group in User Preferences } \\
\text { Database } \\
H: \text { total number of user group } \\
R: \text { there are } R \text { records for one user in } \\
\text { database } \\
W_{G}\left[G R O U P_{b}\right]: \text { user group preferences } \\
W_{P}: \text { personal user preferences } \\
W_{U}: \text { user preferences } \\
\alpha_{3} \quad \beta_{3}: \text { the ratio in user preferences }\end{array}$ \\
\hline
\end{tabular}




\begin{tabular}{|c|c|}
\hline 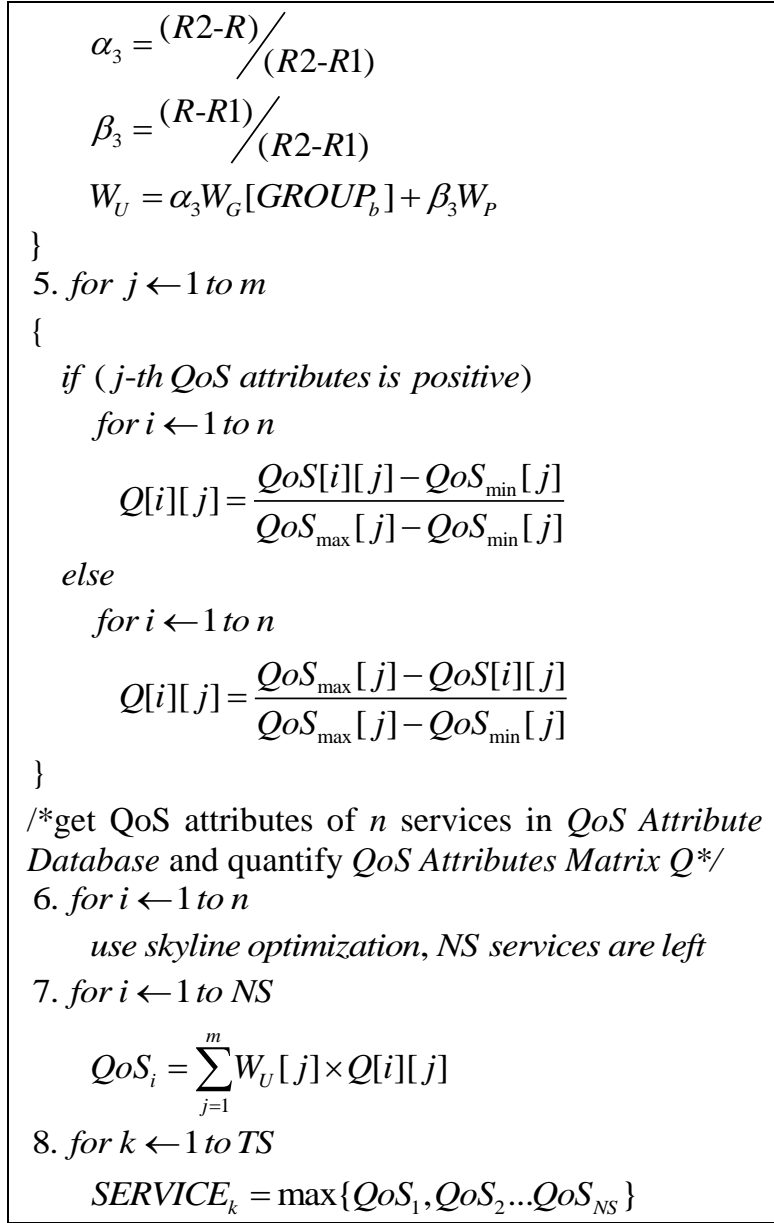 & $\begin{array}{l}\text { Q: QoS Attributes Matrix } \\
\text { NS: the number of service after skyline } \\
\text { optimization } \\
Q O S_{i}: \text { Unified QoS Attributes of service } i \\
\text { SERVICE }_{k} \text { : the selected } k \text {-th service }\end{array}$ \\
\hline
\end{tabular}

Another part of the LBS selection is the updating process in (b). It solves the updating problem of User Preferences Database.

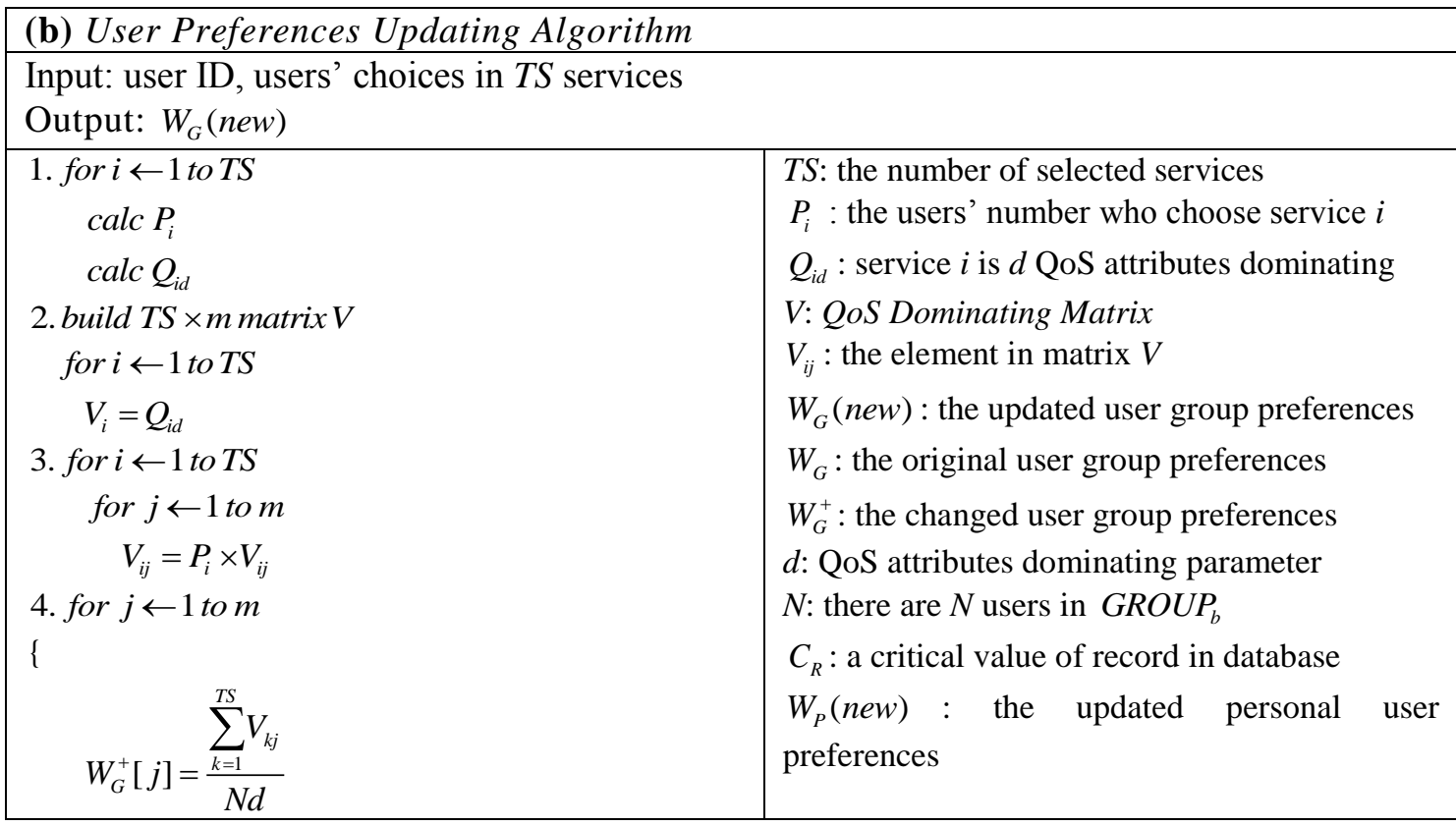




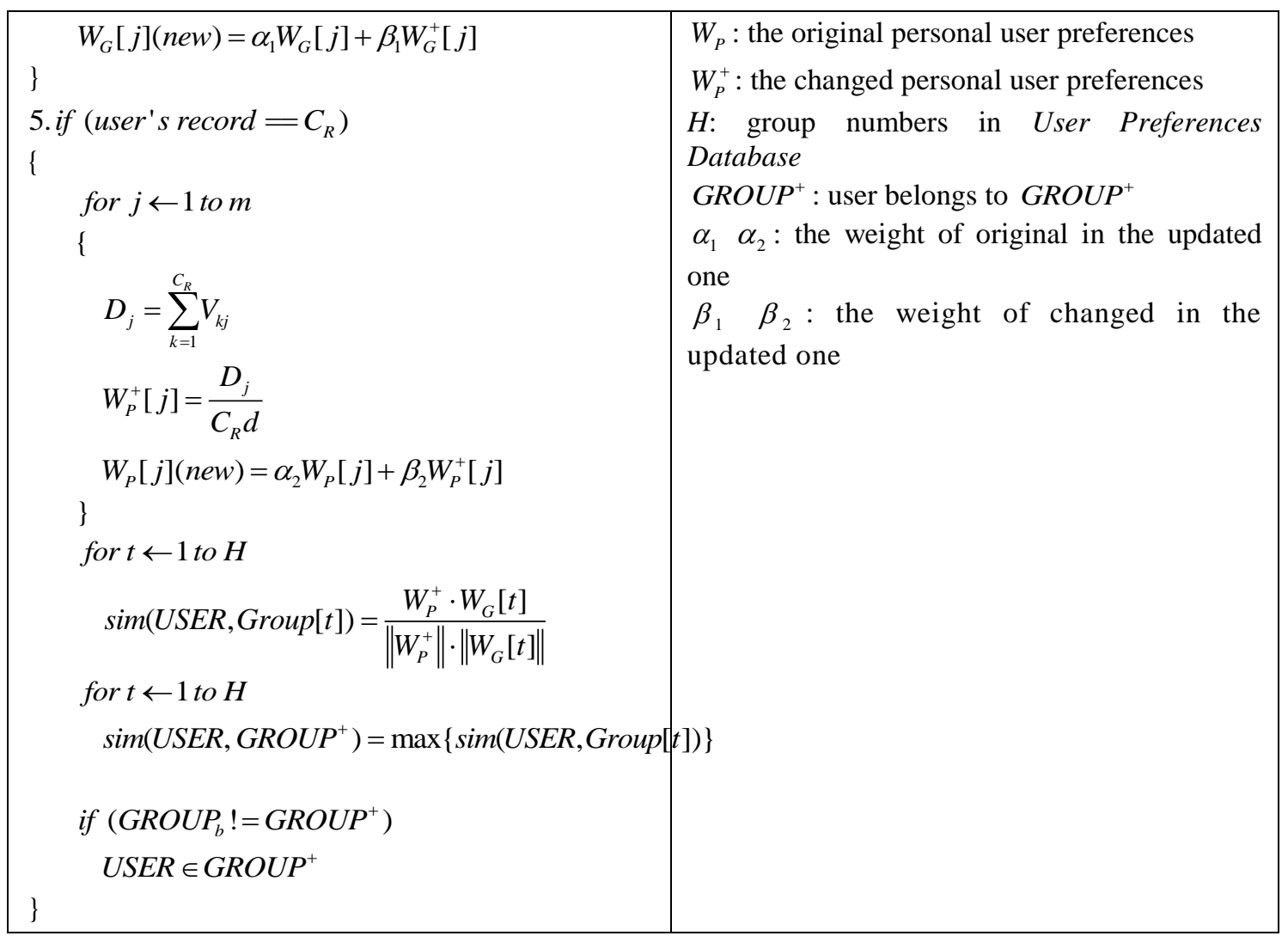

\section{Experiment}

\subsection{Experiment Environment}

To verify the precision and effectiveness of the proposed algorithm in this paper, it designs a Online Learning Resources Platform (OLRP) under the mobile campus environment ${ }^{[14]}$. After one student enters the classroom, he will take a mobile terminal to login the course resources website, using his student ID and password. The system will judge his research direction (which is set as the user preferences) and which course he takes by the time and his location. And then the system will recommend the course resources such as courseware, books and papers. The student will select he likes and then download, recommend and mark. The system records these data as the foundation of the service selection and updating of user preferences.

The foundations of the LBS selection in this experiment are students' research directions (user preferences), students' location and courses they take (location), course resources download times, recommendation times and marking (QoS attributes). A Online Learning Resources Platform (OLRP) has been built in our lab and there are 240 courseware and 100 papers about computer science in $O L R P$. The students can login the website and download what they need for different classes. In this experiment, the LBS selection system is achieved under Android operation system. Figure 2 shows the result of LBS selection. 


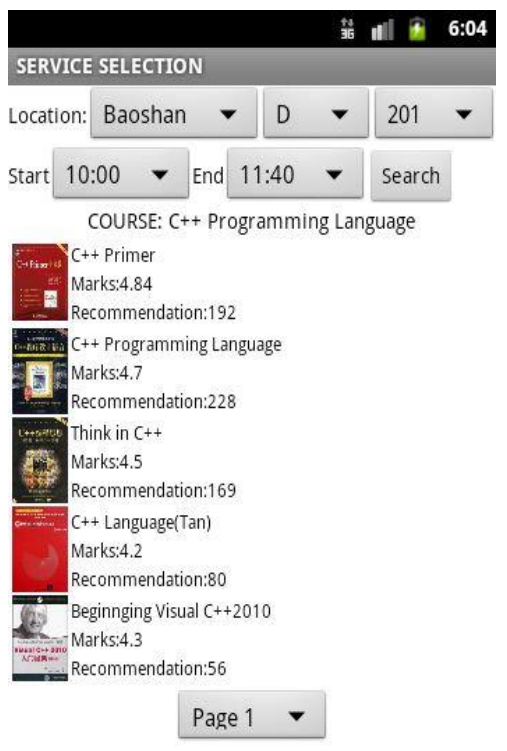

Figure 2. Result of LBS Selection

\subsection{Experiment Index}

Here it gives the definition of experiment index in this service selection.

Download Times: it is the intuitive reflection to the service selection.

Recommendation Times: it reflects the correlation between resources and students, that is the resource conforms the user research direction.

Marking: it means users' evaluation to the resource, ranking from one to five.

In this paper, it uses Rate of Selection to confirm the effectiveness of selection algorithm and it uses Average-QoS and Maximum-QoS as criterion to evaluate the updating algorithm [15].

Rate of Selection

It means how many users will download the resources of service selection.

\subsection{Results of the Experiment}

In the results of service selection, it supposes there are five kind of resources every page in mobile terminal, and if users aren't satisfied with the result, they can turn to page two and find more results.

To calculate the Rate of Selection, two tables are built. These tables are about page weight and item weight in each LBS selection.

Table 1. Values in Each Page

\begin{tabular}{|c|c|c|c|c|c|}
\hline number in each page & 1 & 2 & 3 & 4 & 5 \\
\hline values & 1 & 0.9 & 0.8 & 0.7 & 0.6 \\
\hline
\end{tabular}

Table 2. Values in Each Page

\begin{tabular}{|c|c|c|c|c|}
\hline page & 1 & 2 & 3 & $\cdots \cdots$ \\
\hline values & 1 & 0.5 & 0.25 & $\cdots \cdots$ \\
\hline
\end{tabular}


In Table 1, it means in each page, the Rate of Selection of first item is 1 and the value of third item is 0.8 , and so on. In Table 2, it means the weight of page number in Rate of Selection. For example, 0.5 is the weight of page 2. Formula 10 is used to calculate Rate of Selection.

$$
\text { Rate of Selection }=A(m) \times B(n)=A(m) \times \frac{1}{2^{n-1}}
$$

Here $A(1)=1, A(2)=0.9 \ldots A(5)=0.6$ in Table $1 . n$ means users find what they want in page $n$, and $m$ means this item is the $m$-th in page $n$. If users don't download any resource, then the Rate of Selection is 0 . If users don't make their choices in the service selection process, then the Rate of Selection is also 0.

For example, user downloads resource in third item of page 2. Then the Rate of Selection is $A(3) \times B(2)=0.8 \times 0.5=0.4$.

It uses Rate of Selection to confirm the correctness and effectiveness of service selection. If the Rate of Selection is higher, it means the outputted results satisfy users more. It supposes if there are 100 selection results, then it will calculate the average Rate of Selection ${ }^{[16]}$.

In Figure 3, it is found that as the system running, the Rate of Selection is turning larger. So it means the algorithm is effective and correct to the Online Learning Resources Platform.

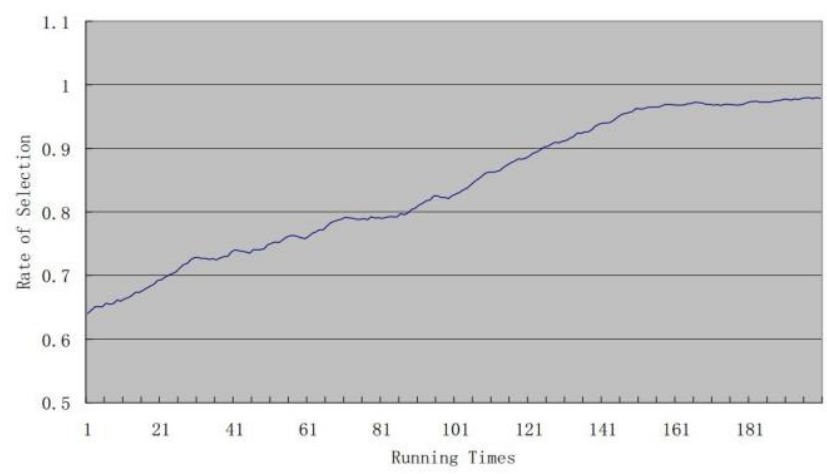

Figure 3. Changing of Rate of Selection

\section{Conclusion}

Nowadays, there are large scales of LBS and applications on the mobile network. So the methods of service selection are necessary as the development of mobile Internet and communication technology. In this paper, it combines the QoS attributes and user preferences, and proposes the mobile service selection model and algorithm based on the QoS constraints and user preferences separately. Furthermore, the result of experiment shows that the algorithm can solve the service selection problem well under mobile Internet environment.

Our ongoing research includes modeling of user preferences with high precision, since the similarity formula in this paper needs to be improved. These services which are selected should satisfy users. That is as the database records more and more users' action, the selection should detect user preferences more correctly. More investigation will be conducted on how to use context information of LBS, such as location and time, and it should concatenate context and user preferences. 


\section{Acknowledgements}

Our research is supported by National Natural Science Foundation of China (Project Number. 61074135) and Shanghai Leading Academic Discipline Project (Project Number.J50103). We should show our great thanks to all of our hard working fellows in the projects above.

\section{References}

[1] J. Feng, "Research on Web Service Selection based on QoS and Reputation", Hunan University of Technology Master's Thesis, (2010).

[2] S.-C. Li, H.-P. Chen and X. Chen, "A Mechanism for Web Service Selection and Recommendation Based on Multi-QoS Constraints”, 2010 IEEE 6th World Congress on Services, pp. 221-228.

[3] Z. Zheng and H. Ma, "QoS-Aware Web Service Recommendation by Collaborative Filtering", IEEE Transactions on Services Computing, vol. 4, no. 2, (2011) April-June, pp. 140-152.

[4] Q., D. and L., "Recommending Social Events from Mobile Phone Location Data", 2010 IEEE International Conference on Data Mining, pp. 971.

[5] K. Virrantaus and J. Veijalainen, "Developing GIS-Supported Location-Based Services", Proc. of WGIS'2001-First International Workshop on Web Geographical Information Systems, Kyoto, Japan, pp. 423-432.

[6] S. Steiniger and Moritz Neun Alistair. Edwardes. Foundations of Location Based Services. University of Zurich.

[7] B. Jiang and X. Yao, "Location-based services and GIS in perspective", Computers, Environment and Urban System, vol. 30, no. 6, (2006), pp. 712-725.

[8] T. D’Roza and G. Bilchev, “An overview of location-based services”, BT Technology Journal, vol. 21, no. 1, (2003), pp. 20-27.

[9] M. F. Mokbel, W. G. Aref and S. E. Hambrusch, "Towards scalable location-aware services: Requirements and research issues", Proceedings of the 11th ACM International Symposium on Advances in Geographic Information System, New Orleans, (2003), pp. 110-117.

[10] M. Shamim Hossain and A. El Saddik, "QoS Requirement in the Multimedia Transcoding Service Selection Process", IEEE Transactions on Instrumentation and Measurement, vol. 59, vo. 6, (2010) June.

[11] ITU-T Recommendation 1.350, General aspects of quality of service and network performance in digital network, including ISDNs. ITU-T, (1993).

[12] B. Krulwich, "Lifestyle finder: intelligent user profiling using large scale demographic data", AI Magazine, vol. 18, no. 2, (1997).

[13] S. Young Jung, J.-H. Hong and T.-S. Kim, “A Statistical Model for User Preference”, IEEE Transactions on Knowledge and Data Engineering, vol. 17, no. 6, (2005) June.

[14] J. Vassileva, "Toward Social Learning Environments", IEEE Transactions on Learning Technologies, vol. 1, no. 4, (2008) October-December.

[15] G. Guo and F. Yu, "A method for semantic web service selection based on QoS ontology", Journal of Computers, vol. 6, no. 2, (2011), pp. 377-386.

[16] C. Liu and D. Liu, "QoS-oriented web service framework by mixed programming techniques", Journal of Computers (Finland), vol. 8, no. 7, (2013), pp. 1763-1770.

\section{Authors}

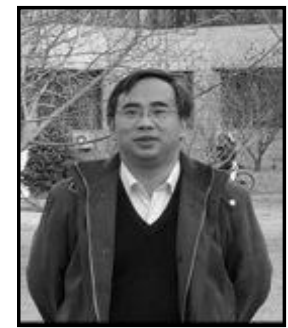

Mingjun Xin is a professor at School of Computer Engineering and Science, Shanghai University. He received his PH.D. from School of Computer Science and Technology, Northwestern Polytechnical University, Xi'an China in 2001. His research interests are Quality of Service (QoS), Location-based Services, mobile platform, service recommendation and software engineering. 


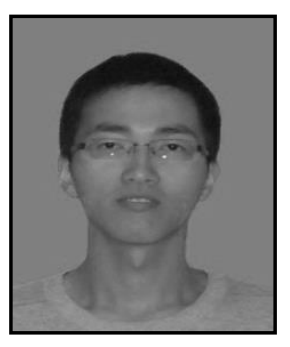

Tianle Jiang is a M.S. student in School of Computer Engineering and Science, Shanghai University. He received his B.S. degree from School of Electronics and Information, Northwestern Polytechnical University, Xi'an, China in 2010. His research interests are Location-based Services, service selection, mobile platforms.

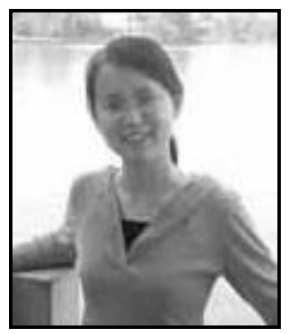

Rui Zhang is a associate professor at School of Computer Engineering and Science, Shanghai University. She received her PH.D from School of Computer Science and Technology, Chinese University of Science and Technology, Hefei China in 2009. Her research interests are Mobile Internet Protocol, Intelligent Mobile Networks, Network Security. 
International Journal of Grid and Distributed Computing Vol.7, No.2 (2014) 